\title{
Prediction of synoptic-scale sea level pressure over the Indian monsoon region using deep learning
}

\author{
Aryaman Sinha, Mayuna Gupta, K S S Sai Srujan, Hariprasad Kodamana $\dagger$, S Sandeep $\dagger$
}

\begin{abstract}
The synoptic-scale (3 - 7 days) variability is a dominant contributor to the Indian summer monsoon (ISM) seasonal precipitation. An accurate prediction of ISM precipitation by dynamical or statistical models remains a challenge. Here we show that the sea level pressure (SLP) can be used as a proxy to predict the active-break cycle as well as the genesis of lowpressure-systems (LPS), using a deep learning model, namely, convolutional long short-term memory (ConvLSTM) networks. The deep learning model is able to reliably predict the daily SLP anomalies over Central India and the Bay of Bengal at a lead time of 7 days. As the fluctuations in SLP drive the changes in the strength of the atmospheric circulation, the prediction of SLP anomalies is useful in predicting the intensity of ISM. It is demonstrated that the ConvLSTM possesses better prediction skill compared to a conventional numerical weather prediction model, indicating the usefulness of a physics guided deep learning model in medium range weather forecasting.
\end{abstract}

Index Terms-ConvLSTM, Sea Level Pressure, Monsoon

\section{INTRODUCTION}

$\mathbf{T}$ HE Indian summer monsoon (ISM) is the key to water security of Southeast Asia, with a population of more than 1.5 billion. An accurate forecast of the ISM rainfall on various temporal and spatial scales is of great importance, from planning for agriculture to disaster preparedness. A dominant mode of ISM rainfall variability, known as synoptic-scale variability, has a temporal scale of 3-7 days and can be extended over the spatial scale of 1000-2000 km [1]. A reliable prediction of synoptic-scale rainfall events is still challenging for conventional dynamical and statistical models [2], [3], [4], [5]. While the data driven models are found to be useful in atmospheric/oceanic predictions, such as El Niño southern oscillation, their skill in ISM rainfall prediction is yet to be proven [6], [7], [8], [9], [10]. The precipitation, being a highly intermittent process, is rather challenging to predict [11]. An easy way to predict the precipitation variability might be to use a proxy variable that is spatially and temporally continuous. Chen and Majda[12] have used outgoing longwave radiation as a proxy to model intraseasonal variability of ISM rainfall. Here, we explore the possibility of using mean sea level pressure (SLP) as a proxy variable to predict the synoptic scale variability of ISM.

The ISM rainfall variability is closely linked to the fluctuations in the intensity of large-scale monsoon circulation

Aryaman Sinha, Mayuna Gupta, and Hariprasad Kodamana (kodamana@iitd.ac.in) are with Department of Chemical Engineering, Indian Institute of Technology Delhi, India. K S S Sai Srujan and S Sandeep (sandeep.sukumaran@cas.iitd.ac.in) are with Center of Atmospheric Sciences, Indian Institute of Technology Delhi, India.

$\dagger$ Corresponding authors. which is driven by the surface pressure gradient [13]. The onset of ISM is also found to be strongly correlated with the surface pressure gradient over India in the month of May [14]. The active and break cycles of ISM are considered as important characteristics of the monsoon [15], [16], [17], [18]. The monsoon breaks have originally been defined on the basis of surface pressure anomalies and later a precipitation based index was developed [17]. Krishnan et al.[16] have identified a northwestward propagating Rossby wave from the Bay of Bengal prior to the onset of the break spells. Such large-scale oscillation patterns indicate an inherent predictability in the active-break cycle of ISM. A 5-6 day oscillation in surface pressure gradient over continental India was reported [19]. In this study, we try to predict the daily mean sea level pressure (SLP) anomalies over the central India with a view to test whether the active-break cycles of ISM can be predicted a week ahead.

The low pressure systems (LPS) which are the synoptic scale storms that form over the Bay of Bengal are responsible for more than half of the seasonal rainfall over the central and northern India [20]. The LPS activity is often associated with the flooding over continental India [21], [22], [23]. The LPS genesis tend to be clustered in the active phase of ISM [24]. As the genesis of LPS is associated with a drop in the SLP, we examine whether the predicted SLP anomalies over the Bay of Bengal give any indication of an LPS formation.

The conventional statistical or dynamical models are known to have limitations in predicting tropical rainfall as it involves a hierarchy of stochastic processes [2], [4]. The deep learning techniques are advancing at fast pace in the recent years and found to be efficient in atmospheric-oceanic predictions [25], [26], [27], [6]. While there have been attempts to predict monsoon onset and regional rainfall using machine learning methods [28], [29], no one has reported prediction of SLP anomalies over monsoon region using deep learning algorithms, to the best of our knowledge. To this extent, we employ a deep convolutional long short-term memory (ConvLSTM) model to predict the SLP anomalies over continental India and the Bay of Bengal at a lead time of seven days during summer monsoon season. ConvLSTM elegantly blends properties of (i) LSTM networks in modeling sequential data, and (ii) CNN in handing spatio-temporal data, and therefore a suitable candidate flexible deep learning model to model SLPA data [6], [30], [31]. We indeed show that, SLP being a continuous variable is more amenable to reliable predictions with inherent predictability and guides as an excellent pointer for identifying active break cycles of ISM. 


\section{DATA AND DEEP LEARNING FRAMEWORK}

The daily SLP data from NCEP/NCAR reanalysis during 1990-2019 is used for developing the ConvLSTM model [32]. Daily SLP anomalies are computed by removing long term mean from the data sets. Further, two regions have been selected - one over the continental India and the second over the Bay of Bengal - to predict SLP anomalies. The region over continental India is bound by $20^{\circ} \mathrm{N}$ to $30^{\circ} \mathrm{N}$ and $70^{\circ} \mathrm{E}$ to $80^{\circ} \mathrm{E}$. The region over the Bay of Bengal is bound by $12^{\circ} \mathrm{N}$ to $22{ }^{\circ} \mathrm{N}$ and $80{ }^{\circ} \mathrm{E}$ to $95^{\circ} \mathrm{E}$. The daily precipitation data at a horizontal resolution of $1^{\circ} \times 1^{\circ}$ from India Meteorological Department (IMD) is also used in this study [33], [34] for validation purposes. The daily precipitation anomalies over Central India $\left(16^{\circ} \mathrm{N}-26^{\circ} \mathrm{N}\right.$ and $\left.74^{\circ} \mathrm{E}-86^{\circ} \mathrm{E}\right)$ are computed by removing the long-term mean. The active (break) spells are defined as three or more consecutive days with precipitation anomalies above (blow) 1 standard deviation of seasonal $\left(1^{\text {st }}\right.$ June to $30^{\text {th }}$ September) precipitation [33]. The monsoon LPS genesis dates were taken from the LPS tracks derived from ERA-Interim daily SLP data by [20] which was also reported by [35]. The ConvLSTM predictions are compared with the SLP from the NCEP-GFS 7-day forecasts for the years 2015 to 2019 , as the public archive of NCEP-GFS forecasts is available from 2015.

The data was organised into training and test sets of 19902010 and 2011-2019, respectively, and area averaged grid values were computed for the test data. Data stacks for training data were prepared in a sliding window fashion for seven day ahead predictions. The overall schematic of the ConvLSTM architecture is shown in Fig. 1. A sequential architecture is used with its first layer as a ConvLSTM 2D layer with ten filters for handling the spatio-temporal training data. The input to the layer is a 5-dimensional tensor having the following attributes: size of training data, channels, latitude, longitude, stack size/time steps. Each input data is a stack of seven frames corresponding to the seven day lagged data for SLP anomalies predictions. The output from this layer is then fed into two blocks consisting of a Conv3D layer having five filters and subsequent batch normalisation layer. A dropout layer is also added to ensure that overfitting of data does not take place during the training phase. Subsequent layers include MaxPooling layers, a flattening layer, and two fully connected dense layers. The salient features of each layer including the number of parameters (trainable + non-trainable) are enlisted in Table I.

For optimisation of the model, the hyper-parameters and layers were tuned to obtain suitable activation, number of filters , optimiser, dropouts, loss functions, epochs, and batch size. The number of epochs were varied from 10 to 500 and the batch sizes were varied 32 to 200 and we obtained satisfactory results with 100-200 epochs and 150-200 batch size during training. A one-third validation split was used on the training data. The best results were obtained with RMSProp optimiser and Rectified Linear Unit (ReLu) activation. Application of both Mean squared error (MSE) loss function and Huber Loss Functions yielded models with good prediction capability.

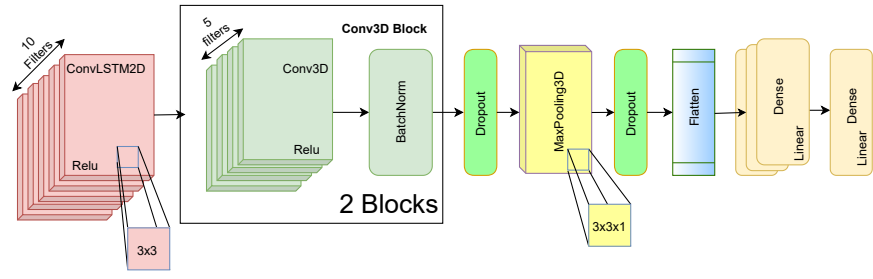

Fig. 1. Deep learning architecture used to predict the sea level pressure anomalies

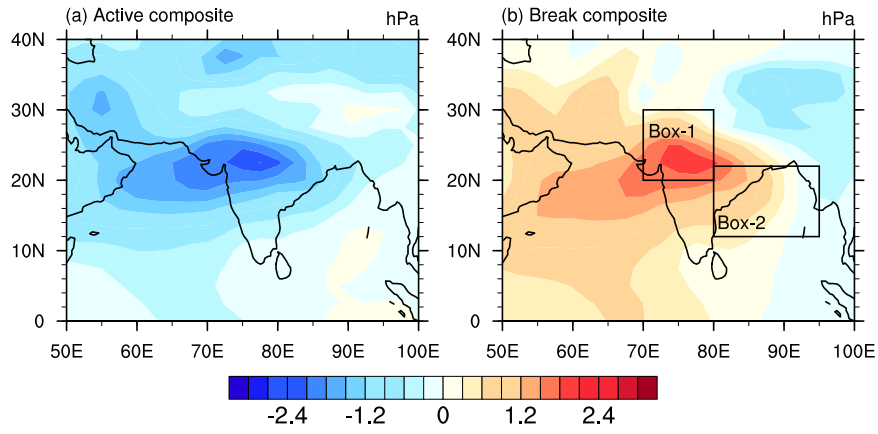

Fig. 2. Composites of sea level pressure anomalies (units: hPa) for (a) active days and (b) break days of Indian summer monsoon during 2000 - 2017. A total of 282 (243) active (break) days were detected during this period. The two boxes in panel (b) show the regions selected to carry out ConvLSTM predictions

\section{RESULTS AND DISCUSSION}

The composites of daily SLP anomalies for active and break monsoon spells during 2000 - 2017 (June - September) period are depicted in Fig. 2. The active composite shows an anomalously low SLP pattern over the Central India, indicating strengthened surface pressure gradient and monsoon circulation (Fig. 2a). The break composite shows an anomalously high pressure over Central India which is associated with weaker meridional pressure gradient and monsoon circulation (Fig. 2b). The daily SLP anomaly predictions are done over two representative regions of ISM - one over continental India and the second over the Bay of Bengal, as shown in Fig. 2b.

Although the ConvLSTM predictions are performed for 2013 - 2019 period, we limit our discussions for four years (2014 - 2017) for brevity. The predicted and observed daily SLP anomaly time series for $1^{\text {st }}$ June to $30^{\text {th }}$ September for four years are shown in Fig. 3. The ConvLSTM predictions of each day are made at a lead time of 7 days. Also, superimposed are the corresponding time series of daily precipitation anomalies. The active and break days are shaded in the precipitation anomaly time series. Overall, the predicted and observed SLP anomalies are in good agreement for all the years, except for slight differences in the amplitude. A closer look at the time series shows that often the predicted SLP anomalies are slightly higher than the observations. A comparison of observed precipitation anomaly time series with the SLP anomalies reveals that the active (break) spells are aligned with the low (high) SLP anomalies. Even if we look beyond activebreak cycles, the low (high) SLP anomalies are associated with the strong (weak) precipitation anomalies throughout 
TABLE I

CONVLSTM ARCHITECTURE DETAILS

\begin{tabular}{|l|l|l|l|l|l|l|}
\hline Layer (type) & Activation & \# Filters & Kernel size & Dropout & Bias & Pool Size \\
\hline ConvLSTM2D & Relu & 10 & $3 \times 3$ & - & - & - \\
Conv3D & Relu & 5 & $3 \times 3 \times 1$ & - & True & - \\
Conv3D-2 & Relu & 5 & $3 \times 3 \times 1$ & 0.2 & True & - \\
MaxPooling3D & - & - & - & 0.2 & - & $3 \times 3 \times 1$ \\
Dense & Relu & 10 & - & - & True & - \\
Dense-2 & Linear & 1 & - & - & True & - \\
\hline
\end{tabular}

TABLE II

BOX 1 \& BOX 2: R2 SCORE AND RMSE OF CONVLSTM V/S GFS

\begin{tabular}{|l|l|l|l|l||l|l|l|l|}
\hline & \multicolumn{4}{|c||}{ Box-1 } & \multicolumn{3}{c|}{ Box-2 } \\
\hline & \multicolumn{2}{|c|}{ convLSTM } & \multicolumn{2}{c|}{ GFS } & \multicolumn{2}{c|}{ convLSTM } & \multicolumn{2}{c|}{ GFS } \\
\hline Year & R2 Score & RMSE & R2 Score & RMSE & R2 Score & RMSE & R2 Score & RMSE \\
\hline $\mathbf{2 0 1 9}$ & 0.93 & 0.84 & -0.16 & 3.43 & 0.83 & 1.15 & -0.40 & 3.26 \\
$\mathbf{2 0 1 8}$ & 0.93 & 0.92 & 0.23 & 2.99 & 0.85 & 1.02 & 0.02 & 2.60 \\
$\mathbf{2 0 1 7}$ & 0.87 & 0.92 & -0.19 & 2.83 & 0.83 & 0.76 & -0.74 & 2.42 \\
$\mathbf{2 0 1 6}$ & 0.87 & 0.85 & -0.74 & 3.16 & 0.69 & 0.97 & -1.31 & 2.65 \\
$\mathbf{2 0 1 5}$ & 0.88 & 1.04 & 0.22 & 3.40 & 0.83 & 0.94 & -0.40 & 2.71 \\
$\mathbf{2 0 1 4}$ & 0.78 & 0.94 & NA & NA & 0.79 & 0.89 & NA & NA \\
\hline
\end{tabular}
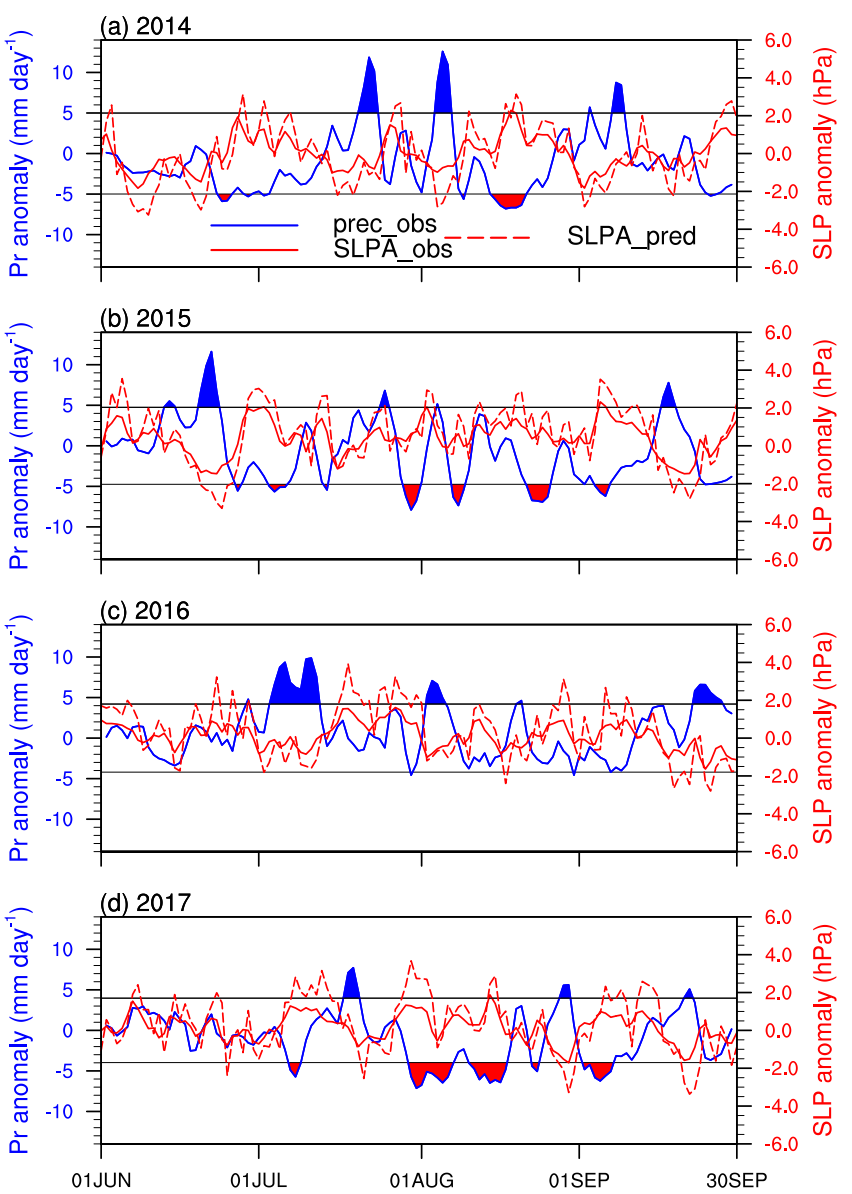

Fig. 3. Time series of observed daily precipitation anomalies, observed sea level pressure anomalies, and predicted sea level pressure anomalies over continental India for the years (a) 2014, (b) 2015, (c) 2016, and (d) 2017 The horizontal lines indicate \pm 1 standard deviation of the daily precipitation anomalies during $1^{\text {st }}$ June to $30^{\text {th }}$ September. The active and break days of monsoon are shaded in blue and red colors, respectively. The sea level pressure anomalies are predicted at a lead time of 7 days. (a) 2014

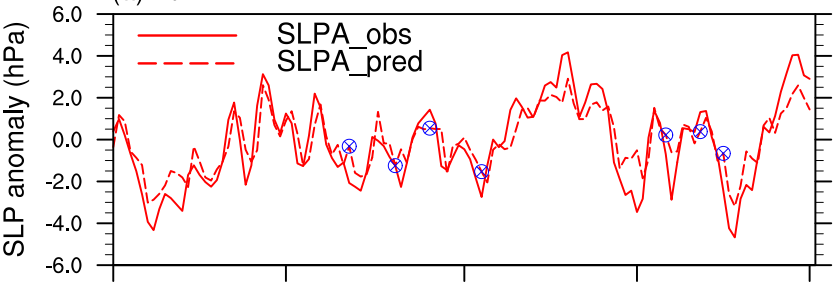

(b) 2015

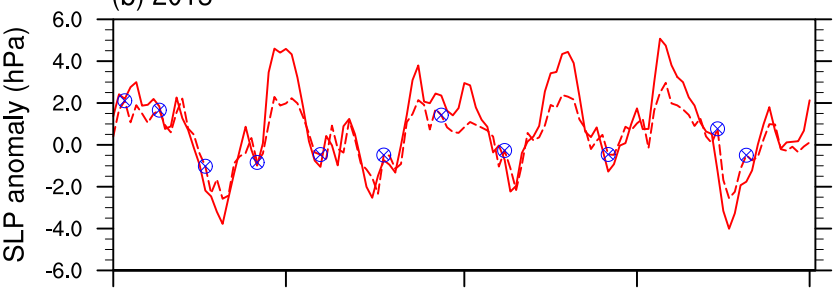

(c) 2016

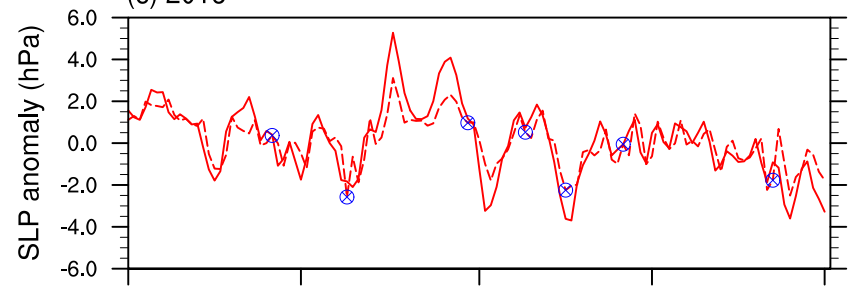

(d) 2017

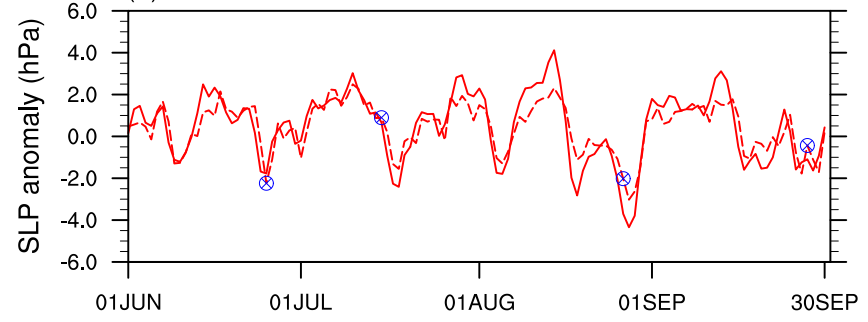

Fig. 4. Time series of observed and predicted sea level pressure anomalies over the head Bay of Bengal for the years (a) 2014, (b) 2015, (c) 2016, and (2017). The markers indicate the dates of genesis of low-pressure systems over the Bay of Bengal. 
the monsoon season. In 2014, two long spells of high SLP anomalies, in June-July and mid-August, are associated with prolonged dry spells (Fig. 3a). A weak wet spell in 2015 seem to be associated with a weak positive SLP anomaly in late July (Fig. 3b). However, other active and break spells of monsoon are all correctly captured by the SLP anomalies. In 2016, there were no break spells over Central India which is in line with the weak positive SLP anomalies in the observations (Fig. 3c). However, the ConvLSTM predicted higher values of positive SLP anomalies, especially during mid-July and late-August. The reason for this overprediction needs to be explored. The longer break spell in August of 2017 is correctly reflected in the high SLP anomalies, though the model overpredicted SLP anomalies in the early part of the break period (Fig. 3d). In general, the SLP anomaly is a good indicator of the active-break phases of ISM over continental India and the ConvLSTM model has reliably predicted the SLP anomalies a week ahead. The realistic prediction of synoptic-scale SLP anomalies by the deep learning model is not surprising as an oscillation with 5-6 day period in SLP is already established [19]. The data-driven models have a good skill in capturing such recurring patterns in complex datasets [26].

The observed and predicted SLP anomaly time series over the Bay of Bengal for the years 2014 - 2017 are shown in Fig. 4a-d. Like the predictions over continental India, the ConvLSTM model has reliably predicted the SLP anomalies over the bay. Interestingly, the model slightly under-predicted the peaks in SLP anomalies compared to the over-prediction over the continental India. As the head Bay of Bengal is the core LPS genesis region, it is important to see if the negative SLP anomalies are giving any indication of storm genesis. The analyses for the four years shown here indicate a genesis of LPS either slightly before a sudden drop in SLP or coincident with the peak negative SLP anomaly. There are also a few exceptions that might be attributed to the differences in the SLP data sets used for ConvLSTM training and tracking of LPS activity.

We compared the performance of the ConvLSTM model with that of a conventional numerical weather prediction model, namely NCEP-GFS model. The RMSE and $\mathrm{R}^{2}$ values of the 7 day ahead SLP predictions by ConvLSTM and NCEPGFS are computed with respect to the corresponding SLP observations for six years (Table II). For the central Indian box, the $\mathrm{R}^{2}$ of ConvLSTM predictions range from 0.78 to 0.93 while that of NCEP-GFS vary from -0.75 to 0.22 , clearly indicating the superior skills of the deep learning model. The RMSE values of SLP predictions by ConvLSTM are in the range of 0.84 to 1 whereas in the case of NCEP-GFS, the RMSE values range from 2.8 to 3.4. Similar statistics are obtained for the SLP predictions over the Bay of Bengal box as well. These statistics confirm that the ConvLSTM model has outperformed the numerical weather prediction model in predicting daily SLP at a lead time of 7 days. It is well known that the forecast quality of the numerical weather prediction models deteriorate over time, due to the errors in the initial conditions as well as the chaotic nature of the atmosphere [36], [37]. Unlike numerical models, the deep learning models are not sensitive to the initial conditions. Also, the deep learning models can perform well when oscillatory signals are present in the data, such as the synoptic scale oscillation in the SLP over India during summer monsoon.

\section{CONCLUSiOnS}

In this study, we demonstrate the prediction of SLP anomalies at a lead time of seven days over two representative regions of ISM using a ConvLSTM model. Although deep learning methods have been successfully used to predict phenomena such as ENSO, their skill in predicting ISM remained ambiguous. The prediction of monsoon precipitation may be difficult, due to the intermittent and stochastic nature of the process. It has long been known that the fluctuations in SLP gradient result in waxing and waning of the monsoon circulation and rainfall. The pressure, being a spatially and temporally continuous variable, is relatively easy to predict by the data driven models. This assumption has been verified by the ConvLSTM predictions of SLP anomalies a week ahead. The apparent relationship between the SLP anomalies and the fluctuations in monsoon rainfall has been clear in our analyses. Our results suggest that the synoptic-scale SLP variability over the continental India can be predicted at least a week ahead. As the SLP anomalies can give an indication of the strength of monsoon rainfall over a broader area, these predictions are useful for planning purposes and have great societal impact. The skill of deep learning model in predicting SLP at a lead time of one week is demonstrated to be better than the skill of numerical weather prediction model. These results suggest that a physics guided deep learning model for ISM rainfall prediction is the way forward.

\section{ACKNOWLEDGMENT}

All the data used in this study are freely available from the following public data repositories. The sea level pressure data is available at https://psl.noaa. gov/data/gridded/ and the daily gridded rainfall data is

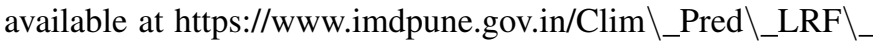
New/Grided \\\_Data \_Download.html.

\section{REFERENCES}

[1] D. R. Sikka, "Some aspects of the life history, structure and movement of monsoon depressions," PAGEOPH, vol. 115, pp. 1501-1529, 1977.

[2] B. Wang, Q. Ding, X. Fu, I.-S. Kang, K. Jin, J. Shukla, and F. DoblasReyes, "Fundamental challenge in simulation and prediction of summer monsoon rainfall," Geophysical Research Letters, vol. 32, no. 15, p. L15711, 2005.

[3] M. Rajeevan, C. Unnikrishnan, and B. Preethi, "Evaluation of the ensembles multi-model seasonal forecasts of indian summer monsoon variability," Clim Dyn, vol. 38, p. 2257-2274, 2012.

[4] B. Wang, B. Xiang, J. Li, P. J. Webster, M. N. Rajeevan, J. Liu, and K. J. Ha, "Rethinking indian monsoon rainfall prediction in the context of recent global warming," Nat Commun, vol. 6, p. 7154, 2015.

[5] S. K. Saha, A. Hazra, S. Pokhrel, H. S. Chaudhari, K. Sujith, A. Rai, H. Rahaman, and B. N. Goswami, "Unraveling the mystery of indian summer monsoon prediction: Improved estimate of predictability limit," Journal of Geophysical Research: Atmospheres, vol. 124, no. 4, pp. 1962-1974, 2019.

[6] M. Gupta, H. Kodamana, and S. Sandeep, "Prediction of enso beyond spring predictability barrier using deep convolutional 1stm networks," IEEE Geoscience and Remote Sensing Letters, pp. 1-5, 2020.

[7] J. Liu, B. Jin, L. Wang, and L. Xu, "Sea surface height prediction with deep learning based on attention mechanism," IEEE Geoscience and Remote Sensing Letters, 2020. 
[8] J. Xie, J. Zhang, J. Yu, and L. Xu, "An adaptive scale sea surface temperature predicting method based on deep learning with attention mechanism," IEEE Geoscience and Remote Sensing Letters, vol. 17, no. 5, pp. 740-744, 2019.

[9] Q. Zhang, H. Wang, J. Dong, G. Zhong, and X. Sun, "Prediction of sea surface temperature using long short-term memory," IEEE Geoscience and Remote Sensing Letters, vol. 14, no. 10, pp. 1745-1749, 2017.

[10] J. Xie, J. Ouyang, J. Zhang, B. Jin, S. Shi, and L. Xu, "An evolving sea surface temperature predicting method based on multidimensional spatiotemporal influences," IEEE Geoscience and Remote Sensing Letters, 2021.

[11] R. Wiuff, "Analysis and modeling of precipitation intermittency by compound markov-darma models," Water Resources Research, vol. 56, no. 2, p. e2019WR025522, 2020.

[12] N. Chen and A. J. Majda, "Predicting the cloud patterns for the boreal summer intraseasonal oscillation through a low-order stochastic model," Mathematics of Climate and Weather Forecasting, vol. 1, no. 1, 2015.

[13] D. R. Sikka, "Some aspects of the large scale fluctuations of summer monsoon rainfall over india in relation to fluctuations in the planetary and regional scale circulation parameters," Proc. Indian Acad. Sci. (Earth Planet Sci.), vol. 89, pp. 179-195, 1980.

[14] A. Chakraborty and S. Agrawal, "Role of west asian surface pressure in summer monsoon onset over central india," Environmental Research Letters, vol. 12, no. 7, p. 074002, 2017.

[15] K. Raghavan, "Break-monsoon over india," Monthly Weather Review, vol. 101, no. 1, pp. $33-43,1973$.

[16] R. Krishnan, C. Zhang, and M. Sugi, "Dynamics of breaks in the indian summer monsoon," Journal of the Atmospheric Sciences, vol. 57, no. 9, pp. $1354-1372,2000$.

[17] S. Gadgil and P. V. Joesph, "On breaks of the indian monsoon," J Earth Syst Sci, vol. 112, pp. 529-558, 2003.

[18] M. R. Ramesh Kumar, R. Krishnan, S. Sankar, A. S. Unnikrishnan, and D. S. Pai, "Increasing trend of "break-monsoon" conditions over india-role of ocean-atmosphere processes in the indian ocean," IEEE Geoscience and Remote Sensing Letters, vol. 6, no. 2, pp. 332-336, 2009.

[19] H. N. Bhalme and S. S. Parasnis, "5-6 day oscillations in the pressure gradients over india during sw monsoon," Indian J. Met. Hydrol. Geophys., vol. 26, no. 1, pp. 77-80, 1976. [Online]. Available: https://metnet.imd.gov.in/mausamdocs/526110.pdf

[20] V. Praveen, S. Sandeep, and R. S. Ajayamohan, "On the relationship between mean monsoon precipitation and low pressure systems in climate model simulations," Journal of Climate, vol. 28, no. 13, pp. 5305-5324, 2015.

[21] V. Krishnamurthy and R. S. Ajayamohan, "Composite structure of monsoon low pressure systems and its relation to indian rainfall," Journal of Climate, vol. 23, no. 16, pp. 4285 - 4305, 2010.

[22] R. Krishnan, D. C. Ayantika, V. Kumar, and S. Pokhrel, "The long-lived monsoon depressions of 2006 and their linkage with the indian ocean dipole," Int. J. Climatol., vol. 31, pp. 1334-1352, 2011.

[23] A. C. Nikumbh, A. Chakraborty, G. S. Bhat, and D. M. W. Frierson, "Large-scale extreme rainfall-producing synoptic systems of the indian summer monsoon," Geophysical Research Letters, vol. 47, no. 11, p. e2020GL088403, 2020.

[24] B. N. Goswami, R. S. Ajayamohan, P. K. Xavier, and D. Sengupta "Clustering of synoptic activity by indian summer monsoon intraseasonal oscillations," Geophysical Research Letters, vol. 30, no. 8, p. 1431, 2003.

[25] P. D. Nooteboom, Q. Y. Feng, C. López, E. Hernández-García, and H. A. Dijkstra, "Using network theory and machine learning to predict el niño," Earth System Dynamics, vol. 9, no. 3, pp. 969-983, 2018. [Online]. Available: https://www.earth-syst-dynam.net/9/969/2018/

[26] M. Reichstein, G. Camps-Valls, B. Stevens, M. Jung, J. Denzler, N. Carvalhais et al., "Deep learning and process understanding for datadriven earth system science," Nature, vol. 566, no. 7743, pp. 195-204, 2019.

[27] Y. Ham, J. Kim, and J. Luo, "Deep learning for multi-year enso forecasts," Nature, vol. 573, p. 568-572, 2019.

[28] A. S. Sahana and S. Ghosh, "An improved prediction of indian summer monsoon onset from state-of-the-art dynamic model using physicsguided data-driven approach," Geophysical Research Letters, vol. 45, no. 16 , pp. 8510-8518, 2018.

[29] R. Sahastrabuddhe, S. Ghosh, A. Saha, and R. Murtugudde, "A minimalistic seasonal prediction model for indian monsoon based on spatial patterns of rainfall anomalies," Clim Dyn, vol. 52, p. 3661-3681, 2019.
[30] X. Shi, Z. Chen, H. Wang, D.-Y. Yeung, W.-K. Wong, and W.-c. Woo, "Convolutional lstm network: A machine learning approach for precipitation nowcasting," arXiv preprint arXiv:1506.04214, 2015.

[31] Y. Bengio, I. Goodfellow, and A. Courville, Deep learning. MIT press Massachusetts, USA:, 2017, vol. 1.

[32] E. Kalnay, M. Kanamitsu, R. Kistler, W. Collins, D. Deaven, L. Gandin, M. Iredell, S. Saha, G. White, J. Woollen, Y. Zhu, M. Chelliah, W. Ebisuzaki, W. Higgins, J. Janowiak, K. C. Mo, C. Ropelewski, J. Wang, A. Leetmaa, R. Reynolds, R. Jenne, and D. Joseph, "The ncep/ncar 40-year reanalysis project," Bulletin of the American Meteorological Society, vol. 77, no. 3, pp. 437 - 472, 1996.

[33] M. Rajeevan, J. Bhate, J. D. Kale, and B. Lal, "High resolution daily gridded rainfall data for the indian region: Analysis of break and active monsoon spells," Current Science, vol. 91, no. 3, pp. 296-306, 2006. [Online]. Available: https://www.jstor.org/stable/24094135

[34] M. Rajeevan, J. Bhate, and A. K. Jaswal, "Analysis of variability and trends of extreme rainfall events over india using 104 years of gridded daily rainfall data," Geophysical Research Letters, vol. 35, no. 18, p. L18707, 2008.

[35] M. Meera, E. Suhas, and S. Sandeep, "Downstream and in situ: Two perspectives on the initiation of monsoon low-pressure systems over the bay of bengal," Geophysical Research Letters, vol. 46, no. 21, pp. 12 303-12 310, 2019.

[36] W. Duan and C. Wei, "The 'spring predictability barrier' for enso predictions and its possible mechanism: results from a fully coupled model," International Journal of Climatology, vol. 33, no. 5, pp. 12801292, 2013.

[37] M. Mu, H. Xu, and W. Duan, "A kind of initial errors related to "spring predictability barrier" for el niño events in zebiak-cane model," Geophysical Research Letters, vol. 34, no. 3, 2007. 